Autism

The International Joumal of Research and Practice

\title{
Change detection of meaningful objects in real-world scenes in adolescents with and without ASD
}

\begin{tabular}{|c|c|}
\hline Journal: & Autism \\
\hline Manuscript ID & AUT-16-0312.R1 \\
\hline Manuscript Type: & Original Article \\
\hline Keywords: & $\begin{array}{l}\text { Autism spectrum disorders, Vision research, Change blindness, Local-global } \\
\text { processing }\end{array}$ \\
\hline Abstract: & $\begin{array}{l}\text { Previous research suggested that adolescents with Autism Spectrum } \\
\text { Disorder (ASD) are better than Typically Developing (TD) children in } \\
\text { detecting local, non-social details within complex visual scenes. To better } \\
\text { understand these differences, we used the image database by Sareen and } \\
\text { colleagues (2015), containing the size and on-screen location information } \\
\text { of all changes in the images, in a change blindness paradigm. In this task } \\
\text { an original and a modified real-world scene, separated by a grey blank, } \\
\text { alternate repeatedly until observers detect the change. Our results } \\
\text { indicated that participants with and without ASD performed similarly when } \\
\text { scenes were presented upright, but that only the performance of the TD } \\
\text { adolescents became worse in the inverted scene condition. In this } \\
\text { condition, the correlation between performance and both image difficulty } \\
\text { and change predictability was significantly weaker in ASD than in TD } \\
\text { participants. We suggest that these findings result from a more locally } \\
\text { biased search strategy in people with ASD, compared to TD participants, in } \\
\text { tasks in which the rapid processing of global information does not help to } \\
\text { improve change detection performance. Finally, although we found change } \\
\text { location, change size and age to influence participant performance, none of } \\
\text { these was directly linked to the observed group-level differences. }\end{array}$ \\
\hline
\end{tabular}

\section{SCHOLARONE" Manuscripts}




\title{
Change detection of meaningful objects in real-world scenes
}

\author{
in adolescents with and without ASD
}




\begin{abstract}
Previous research suggested that adolescents with Autism Spectrum Disorder (ASD) are better than Typically Developing (TD) children in detecting local, non-social details within complex visual scenes. To better understand these differences, we used the image database by Sareen and colleagues (2015), containing the size and on-screen location information of all changes in the images, in a change blindness paradigm. In this task an original and a modified real-world scene, separated by a grey blank, alternate repeatedly until observers detect the change. Our results indicated that participants with and without ASD performed similarly when scenes were presented upright, but that only the performance of the TD adolescents became worse in the inverted scene condition. In this condition, the correlation between performance and both image difficulty and change predictability was significantly weaker in ASD than in TD participants. We suggest that these findings result from a more locally biased search strategy in people with ASD, compared to TD participants, in tasks in which the rapid processing of global information does not help to improve change detection performance. Finally, although we found change location, change size and age to influence participant performance, none of these was directly linked to the observed group-level differences.
\end{abstract}




\section{Introduction}

People often fail to notice large changes to visual scenes, a phenomenon often referred to as change blindness (for review, see Simons and Ambinder, 2005). In a typical change blindness experiment, an original and a modified real-world scene, separated by a brief blank display, alternate repeatedly until observers discover the change. Previous research in Typically Developing (TD) participants indicated that focused attention on the location of the change is required to detect the change (Rensink et al., 1997). This representation of local stimulus elements is effortful and contrasts with the extremely rapid capture of the conceptual, global, gist of a scene (Hochstein and Ahissar, 2002). Consistently, TD participants were found to report the overall statistical properties of a stimulus set more easily than the individual properties (Ariely, 2001). More precisely, these authors administered both a member identification task (e.g., whether an individual item of a particular size was presented in a set of circles with variable size) and a mean discrimination task (e.g., whether an individual item of a particular size was smaller or larger than the average size of a set of circles) to TD participants. They found that TD observers knew little or nothing about the size of the individual items in a set, while they could encode quite precise information about the mean of the set. Based on these results, the authors proposed that the visual system automatically creates a representation of the global properties of the whole set when presented with a set of 
multiple similar items and discards the information about the individual items (or details) in this set. This interpretation is in line with the idea that the visual processing of a stimulus (or a scene) starts with the parallel extraction of different visual attributes at different spatial frequencies following a predominantly coarse-to-fine default processing sequence (e.g., Bullier, 2001; Kauffmann et al., 2014). More precisely, our initial scene perception is based on widely distributed attention identifying 'the forest before the trees', while later vision focuses attention to details in the display. This could explain why detecting change is so difficult for TD participants and why the method has the potential to increase our understanding of the attentional preferences of people with Autism Spectrum Disorder (ASD), who tend to be less globally and more locally biased when processing visual information, especially when local incongruent information is present in the stimulus (Van der Hallen et al., 2015).

A number of papers have appeared, describing change detection abilities in ASD, either arguing for or against theories that emphasize the local processing bias or advantage often apparent in people with ASD (Ames and FletcherWatson, 2010). However, findings remain mixed and often inconsistent (FletcherWatson et al., 2012; Maccari et al., 2014). For example, some studies reported people with ASD to be equally susceptible as TD participants to the semantic relevance and the contextual importance of a change during change blindness (Burack et al., 2009; Fletcher-Watson et al., 2006). The semantic relevance of an item is thereby defined as 
the meaning or role attributed to an item in the visual array, and the contextual importance refers to the relationship between the change and its surroundings. More precisely, all participants, with and without ASD, performed similarly and detected changes to semantically meaningful and contextually inappropriate items faster and more accurately than to less semantically important and appropriate objects. However, in contradiction with these findings, another study reported a complete lack of contextual sensitivity in people with ASD (Loth et al., 2008). While the TD participants detected scene-unrelated changes significantly better than scene-related substitutions, the people with ASD were not influenced by scene consistency. This resulted in a significantly slower and less accurate detection of scene-unrelated objects by the people with ASD, which was interpreted as a reduced influence of schematic expectations on the spontaneous attention in individuals with ASD and evidence against a local processing advantage in ASD. Finally, to complicate things further, there are also studies that observed an enhanced performance of participants with ASD, compared to TD participants, with respect to the perception of objects embedded within a complex visual display (Fletcher-Watson et al., 2012; Smith and Milne, 2009). In general, it has been suggested that people with ASD performed worse than TD participants when the change involves socially relevant (facial) information (Kikuchi et al., 2009), while outperforming them in detecting less meaningful (incongruent) changes (Smith and Milne, 2009). This large discrepancy in study outcomes could be explained by a large 
within-population heterogeneity in ASD (e.g., differing ages, sex distributions, intellectual ability,...) and differences in experimental methodology (e.g., open-ended versus forced-choice tasks, complex versus simple content, moving versus static stimuli,...) (Fletcher-Watson et al., 2012; Maccari et al., 2014). In order to overcome these problems, the current study carefully matched participant groups with and without ASD on baseline characteristics (IQ, age and gender) and aimed to systematically assess whether or not adolescents with ASD were indeed faster than TD adolescents, in observing local changes in visually complex, non-social scenes. This was done by carefully examining which stimulus conditions were especially important for influencing this performance. Interestingly, our study is the first (to our knowledge) to focus on the influence of scene inversion (upright versus inverted) in a non-social change blindness paradigm in people with ASD.

Scene inversion disrupts the extraction of meaning and alters the semantic evaluation of objects and scenes (Epstein et al., 2006 ; Shore and Klein, 2000). While previous findings in TD participants indicated that changes to semantically central items are detected faster than more marginal changes (Rensink et al., 1997; Smith and Milne, 2009), even when controlling for the visual saliency of these changes, scene orientation significantly reduced this detection advantage (Kelley et al., 2003). The authors interpreted this finding by assuming that scene inversion disrupts or slows down gist perception, translating in a less efficient deployment of attention and change detection 
performance. Furthermore, since the low-level perceptual differences between an upright and corresponding inverted image are minimal (e.g., equally bright, the same contrast profiles, colors,...), this inversion effect is assumed to be associated with removing the upright image from its original context, that is, shattering configural processing or holistic orientation (Rakover and Cahlon, 2001). As a result, the increased attention for processing local stimulus elements or features in ASD, focusing less on extracting the semantic (global) gist of the scene, could be an advantageous strategy when detecting changes in meaningful inverted scenes (Koldewyn et al., 2013; Mottron et al., 2006). However, this detail-focused processing of visual information might simultaneously be disadvantageous when detecting changes in the upright presented scenes. More precisely, in upright presented scenes TD participants will be able to rapidly interpret the overall gist of the scene and to use this information to efficiently search the display to detect task-relevant stimulus changes. As a result, we assumed that participants with ASD would only perform worse than their TD counterparts when the implemented changes in the upright presented scenes would be semantically meaningful (and, therefore, imply a bidirectional relation between the local change and the scene gist). Inverting the scene would then eliminate the global processing advantage of TD participants. However, given the default coarse-to-fine processing sequence of visual information in TD participants versus the more detailoriented perception of people with ASD (Mottron et al., 2006), even when changes 
would be less semantically meaningful, we might still observe a change detection advantage in ASD for inverted scene information. More precisely, the increased attention for local scene elements in people with ASD might minimize, compared to TD participants, the disruptive effect scene inversion has on their change detection performance. Furthermore, we reasoned that this more detail-focused processing in ASD would also be linked to a stronger reliance on more low-level physical stimulus information during change detection. More specifically, we focused on two possibly important variables of interest: (a) the location, and (b) the size of the change.

Change location. Objects in a scene that preferentially receive attention are more likely to be encoded (Simons and Rensink, 2005) and TD observers more often report the scene alteration when this change occurred closer to the fixation location of the eyes at the moment of change detection (O'Regan et al., 2000). In the current research, we focused on the distance of the changes to the physical center of the image. Using this manipulation of change location, no apparent effect was found in the recent change blindness study of Sareen and colleagues (2015) in TD participants. Interestingly, in the current study we also assessed the perceived predictability (low versus high) and semantic centrality (central versus marginal) of the changes in the images by testing an independent group of TD raters. This was done to assess possible group-level differences in change detection strategy between people with and without ASD. More precisely, we used the TD ratings of subjective predictability and semantic 
centrality as a baseline measure, and correlated it with the change detection performance of both the adolescents with and without ASD. We thereby reasoned that possible group-level differences in correlation strength would reflect differences in the (expectation-based) use of global gist information to locate the change within the scene. This would be in line with previous research suggesting that, although people with and without ASD were found to detect semantically central changes better than more marginal changes, people with ASD detected more marginally located changes slower than their TD counterparts (Fletcher-Watson et al., 2006). The authors explained this difference based on a difficulty to switch attention between the items in the scene, leading to a slower search for the locus of the change. Nonetheless, these findings directly contradicted with a more recent study indicating that participants with ASD detected significantly more changes than the TD participants, especially due to a better detection of more marginal changes in the scene (Smith and Milne, 2009). A possible explanation of these different outcomes might be that the former study (FletcherWatson et al., 2006) used an alternating static image display, while the latter study (Smith and Milne, 2009) was based on the detection of continuity errors in a movie and required a deeper level of processing (e.g., asking multiple questions at once). In the current research, using a static image display, we hypothesized that all participants would be better at detecting more meaningful, compared to more marginal, changes in the upright scene condition when the implemented changes were regarded as 
semantically meaningful within the different scene contexts (similar to Kelley et al., 2003). Furthermore, we also predicted that TD participants would perform better than people with $\mathrm{ASD}$, when the rapid extraction of gist information could be used to efficiently search the display to detect task-relevant stimulus changes. We did not expect this context effect to be present in the inverted scene condition.

Change size. No apparent effect of the size of the change, measured in terms of pixel area, was found in the recent change blindness study of Sareen and colleagues (2015) using their indoor scenes database. Nonetheless, given that change detection performance in TD participants is correlated with the distance between the changing region and the point of fixation immediately before or after the change (Henderson and Hollingworth, 1999; O'Regan et al., 2000), we predicted that larger changes would be observed faster than smaller alterations, given the larger probability of these changes to be in the vicinity of an eye fixation. Given that we are exclusively using non-social scenes in the current study, and atypical eye-movement patterns in people with ASD are mainly reported for the processing of social information (Senju, 2013), we did not expect differences in the attentional pattern of people with and without ASD in the upright scene condition. However, in the inverted scene condition, non-semantic image properties, such as (low-level) object size information, might have a stronger influence on change detection performance. To test this assumption further in the current study, we also correlated our behavioral findings on the change detection task with measures 
of non-semantic image properties. The first is low-level visual saliency (for details, see Walther and Koch, 2006), which can be defined as the extent to which an item or an object in a display automatically draws the attention of the observer. Previous research thereby indicated that people with ASD generally attend faster to low-level salient information (e.g., pixel-level color, intensity, orientation) than TD participants, while attending slower to semantic-level saliency (e.g., contextually relevant objects) (Wang et al., 2015). Therefore, although we believed that all participants would be guided more by low-level visual saliency in the inverted scene condition (in which the extraction of meaning is disrupted), we expected that this would be more the case for adolescents with ASD than for their TD counterparts. Furthermore, we also examined non-semantic properties extracted from the Pyramid Histogram of Oriented Gradients (PHOG): selfsimilarity, complexity and anisotropy (for details, see Braun et al., 2013). The property of self-similarity implies that an image as a whole has an appearance similar to its parts (e.g., a tree having the same structure as one of its branches, having the same structure as the veins of its leaves). Complexity is defined as the irregularity or heterogeneity of a pattern (e.g., the number of different elements that form the scene) and anisotropy as the lack of uniformity in directionality or orientation of image features (e.g., edges distributed more vertically or horizontally). Similar as for low-level visual saliency, we predicted that these variables would be more strongly correlated with the performance of our participants in the inverted scene condition. 


\section{Methods and materials}

\section{Participants}

A group of 26 adolescents ( 22 male, 4 female) with ASD (mean age $=13.89 ; S D=1.53$; [min $\max ]$ age $=[12$ 17]; Inter Quartile Range $(I Q R)=2)$ and a similarly sized TD control group (mean age $=14.35 ; S D=1.23 ;[\min \max ]$ age $=[1317] ; I Q R=2)$, which were individually matched on age, gender and IQ, participated in this study (see Table 1 for participant characteristics). IQ was estimated using an abbreviated four-subtest (Vocabulary, Similarities, Picture Completion and Block Design) version of the WISCIII (Sattler, 2001; Wechlser, 1997). All participants also completed the Dutch Social Responsiveness Scale (SRS) questionnaire (Roeyers et al., 2011) to get an overall estimation of individual and/or group-level differences in ASD traits.

Participants from the ASD group were previously diagnosed with a pervasive developmental disorder (Autistic Disorder, Asperger syndrome or PPD-NOS), according to DSM-IV-TR criteria (American Psychiatric Association, 2000), by a multidisciplinary team. Recruitment was exclusively set up via the Autism Expertise Centre of the University Hospital in Leuven. Furthermore, a trained clinical psychologist administered the Dutch version of the Autism Diagnostic Observation Schedule 2 (ADOS-2) module 3 (Gotham et al., 2006; Dutch version: de Bildt et al., 
2009) from all participants with a clinical diagnosis and ASD diagnoses were reconfirmed in 24 of the 26 adolescents, with the new ADOS Algorithm for DSMIV/ICD-10 (ADOS-2). Since the analyses did not differ depending on whether we in- or excluded the participants scoring below the ADOS-2 cut-off score, we followed the clinical diagnosis of the participants and reported the results of the full ASD group. All participants had normal or corrected-to-normal vision. The study was approved by the Medical Ethics Commission of KU Leuven and both the participants themselves and their parents provided written informed consent before onset of the experiment.

\section{Stimuli and procedure}

Participants were seated at $57 \mathrm{~cm}$ from the calibrated (gamma corrected) computer monitor (resolution: 1920 x 1200; refresh rate: $60 \mathrm{~Hz}$; type: Monitor DELL U2410) in a dimly lit room. The head position of the participants was stabilized by means of a head and chin rest and we used 110 colored images (1024 x 768 pixels) from the indoor scenes database by Sareen and colleagues (2015). The images in the short practice sessions (10 images) were different from those in the testing phase (2 sets of 50 images). Each participant had to respond, during the testing phase, to either the first or the second set of 50 images. Each scene could be left-right reversed and presented upright or inverted. Nonetheless, each participant saw only one version of the same 
scene. Some examples of the stimuli are shown in Figure 1B. All images can be found on http://gestaltrevision.be/en/resources/supplementary-material.

On each trial a fixation cross (apparent size: $1 \times 1^{\circ}$ ) appeared for $1000 \mathrm{~ms}$, followed by a briefly presented $(240 \mathrm{~ms})$, meaningful color picture (apparent size: $27 \mathrm{x}$ $20.5^{\circ}$ ). This image was followed by a blank (grey background; $240 \mathrm{~ms}$ ), an altered image (240 ms) and another blank (240 ms). Such an alternation, between an image and its modified version, kept repeating itself until the participant pressed a response. This response was based on the task instructions, which specified that the participants had to indicate as fast as possible, but without guessing, where (left or right side) the change in the image took place, using the left and right arrow on the keyboard. To avoid confusion between left and right changes closer to the center of the image, on each trial a black reference line (outside the image frame) clearly highlighted the middle of the stimulus and all changes objectively occurred clearly to the left or right side of the image center. A change was present in all trials and half of the scenes were altered on the left, and the other half on the right side, of the image. Each trial either ended because the observer pressed the left/right arrow key to confirm the presence of a change or, if the participant was not able to observe the change in the scene, after 40 seconds of unresponsiveness. These time-out trials were regarded as incorrect responses in the analysis and, given the explicit instruction not to guess, almost all incorrect responses in the task were time-out trials. Before starting, participants completed a practice session with visual trial-by-trial 
feedback (a green/red fixation cross after each correct/incorrect response, respectively) to familiarize them with the design. During these practice trials, participants were clearly instructed by the experimenter not to guess and only to answer when they actually saw the change in the presented scene. During the actual experiment no feedback was provided.

Furthermore, in a separate experiment, we asked an independent group of TD participants $(n=30)$ to rate the predictability (How predictable is the change in the scene?) and the semantic centrality (How meaningful is the change in the scene?) of each of the changes, in each of the images, on a 7-point Likert scale. Lower values thereby indicated less and higher values more perceived predictability/semantic centrality of the change. To complete this task, both the original and the altered version of each image were shown simultaneously, and without time constraints, to the participants. If they were not able to locate the change in the scene themselves, they were allowed to ask for a 'clue' (which was not included in the main experiment). This 'clue' unambiguously illustrated the exact location of the change (for an overview, see Supplementary materials). We preferred independent participants for these ratings because we did not want the ratings to be influenced by the change detection task or vice versa. 


\section{Analyses}

We calculated the median reaction time (RT) and mean accuracy performance for each of the participants as a dependent variable in a mixed ANOVA with Group (TD versus ASD) as between-subjects factor and scene inversion (upright versus inverted) as within-subjects factor. Participants were regarded as a random factor.

We then analyzed the trial-by-trial response RT and accuracy (correct/incorrect) scores as the dependent variables in a General Linear Mixed Modeling (GLMM) approach (McCullagh, 1984), to account for the influence of change location and size on participant performance. After model selection, for both dependent variables separately, the individual predictive value of each selected parameter was tested using (1) Welch's t-test with Satterthwaite approximation for the denominator degrees of freedom (McArdle, 1987) in the random intercepts regression analysis for RT and (2) Wald Z-tests (Wald, 1943) in the random intercepts logistic regression analysis for accuracy. Participant was regarded as random intercept and participant characteristics (FSIQ and Age (in years) as continuous variables and Gender (boy versus girl) as a dichotomous variable) were added as possible covariates to the GLMM analysis.

Finally, we calculated the bilateral Pearson correlation coefficients between the mean RT scores per image, for the group-level specific performance (ASD and TD) in the upright and inverted condition separately, and (1) the mean RT performance per image in the study by Sareen et al. (2015) as a measure of image difficulty, (2) the X 
(horizontal) and Y (vertical) pixel location of the center of the change, (3) the visual saliency of the change, operationalized as the predicted number of fixations of a human observer preceding a saliency peak at the location of the change (for details, see Walther and Koch, 2006), (4) the rated predictability, (5) the rated semantic centrality of the changes, (6) whether or not a clue was needed to observe the change in the rating task, and (7) measures of non-semantic (global) image properties such as self-similarity, complexity and anisotropy (for details, see Braun et al., 2013). If meaningful correlations were observed, we used Steiger's Z test to test whether the strength of these correlations differed between the different conditions (Steiger, 1980).

All outcomes were obtained by using the statistical software program $\mathrm{R}$ version 3.1.1 (R core team, 2013), the MATLAB Saliency Toolbox 2.3 (Walther and Koch, 2006) and IBM SPSS (version 22).

\section{Results}

\section{Mixed ANOVA}

There was no significant main effect of Group on participant performance. The analysis did provide evidence for a significant $\mathrm{RT}$ main effect of Scene inversion (RT: $F_{1,50}=$ 9.45; $\left.p<.01 ; \eta^{2}=.16\right)$ and a significant RT Scene inversion * Group interaction effect $\left(\mathrm{RT}: F_{1,50}=3.92 ; p=.05 ; \eta^{2}=.07\right)$. More precisely, inverting the scene made change 


\begin{abstract}
detection slower for all participants, but this effect was significantly more pronounced for TD adolescents than for adolescents with ASD (Figure 2).
\end{abstract}

\title{
GLMM analysis
}

In accordance with the mixed ANOVA, we did not observe a significant main effect of Group on participant performance in the GLMM analysis (for parameter estimates and $95 \%$ confidence intervals, see Supplementary materials). This indicated that the detection of changes in indoor scenes, presented upright, lead to similar results in adolescents with and without ASD.

Scene inversion (Figure 2). The final model of the GLMM analysis, modeling RT, only contained a significant Group * Scene inversion interaction effect $\left(t_{38.89}=\right.$ $3.45 ; p<.001$ ), but no significant main effect of Scene inversion. This could indicate, in line with the graphical illustration in Figure 2, that scene inversion only hampered change detection in TD adolescents, while not significantly affecting performance of adolescents with ASD.

Change location. Our results indicated an overall main effect of change location (RT: $t_{39.52}=-3.63 ; p<.001 \mid$ Accuracy: $\left.Z=5.82 ; p<.001\right)$. This finding provided evidence for a faster and more accurate detection of less centrally located changes within the scenes. No interaction between change location and group-level performance was observed. 
Change size. Our findings also pointed towards a significant main effect of the change size (RT: $\left.t_{38.88}=-3.24 ; p<.01\right)$ : larger changes were detected faster than smaller changes by all adolescents. No interaction between change size and group-level performance was observed.

Other predictors of performance. Our results also indicated a significant main effect of Age (RT: $t_{51.70}=-2.86 ; p<.01 \mid$ Accuracy: $\left.Z=2.71 ; p<.01\right)$ and Image order (RT: $t_{38.99}=-3.77 ; p<.001 \mid$ Accuracy: $Z=1.96 ; p=.05$ ). While the former effect implied that older adolescents conducted the change detection task both faster and better than their younger counterparts, the latter observation could be explained by a general learning effect across trials, implying that all participants became faster during the task.

\section{Correlation analysis (Table 2)}

Image difficulty. We found significant, negative, correlations for all conditions (ASD upright, ASD inverted, TD upright, TD inverted) between RT and our measure of image difficulty (Figure 3A). Interestingly, this correlation was stronger in the upright than in the inverted condition, both for $\operatorname{ASD}(Z=2.15 ; p=.03)$ and $\operatorname{TD}(Z=2.07 ; p=$ .04) participants. Furthermore, in the inverted condition, the correlation was significantly smaller for the ASD, compared to the TD group $(Z=2.38 ; p<.01)$. 
Semantic centrality. Although all correlations with our measure of semantic centrality were in the expected (negative) direction, no significant correlations were found (Figure 3B).

Predictability. We found significant, negative, correlations for all conditions and our measure of predictability (Figure 3C). Interestingly, for the $\operatorname{ASD}(Z=1.95 ; p=$ $.05)$, but not the $\operatorname{TD}(Z=1.70 ; p=.09)$ group, this correlation was stronger in the upright than in the inverted condition. Furthermore, in the inverted condition, the correlation was significantly weaker for the ASD, compared to the TD, group $(Z=1.93$; $p=.05)$

Clue needed in the rating task. We found significant, positive, correlations for all conditions and the proportion of people in the independent group of TD raters requesting a 'clue' to find the change in the rating task (Figure 3D). Interestingly, this correlation was stronger in the upright than in the inverted condition, both for $\operatorname{ASD}(Z=$ $2.27 ; p=.02)$ and $\operatorname{TD}(Z=2.89 ; p<.01)$ participants. Furthermore, in the inverted condition, the correlation was significantly weaker for the ASD, compared to the TD, $\operatorname{group}(Z=1.94 ; p=.05)$

Other correlations. No other significant correlations were found, nor did these observations change when explicitly controlling for the image change location and/or the change size. 


\section{Discussion}

In the current study, we systematically assessed whether or not adolescents with ASD performed differently in detecting local changes of complex visual scenes using a change blindness paradigm. Our findings indicated that all participants were able to effectively complete the task and that no differences in performance were observed between adolescents with and without ASD in the upright scene condition. Nonetheless, given the large heterogeneity in the findings of previous studies on change blindness in ASD (e.g., Fletcher-Watson et al., 2012; Maccari et al., 2014), our findings have to be interpreted with caution. One important factor contributing to the similar performance of people with and without ASD might be that our image set only contained contextually-relevant changes, while group-level differences were previously reported to result from an enhanced (or less contextually influenced) perception of incongruent scene elements (Loth et al., 2008; Smith and Milne, 2009). This suggested an abnormally broad attentional spotlight and enhanced perceptual functioning in individuals with ASD, possibly due to an inefficient filtering of visual information. The absence of congruency errors in the current image set might therefore leave the rapid global gist extraction in ASD intact, helping them to rapidly focus attention on the details of the image (as people with ASD might do instantaneously). Furthermore, the changes were generally rated as being more semantically marginal 
than central to the given scene contexts. As the a posteriori correlation analysis indicated, this resulted in the absence of a significant influence of change meaningfulness on detection performance. This could imply that rapid gist interpretation did not provide a strong advantage for detecting local changes in the current change blindness paradigm.

Interestingly, our findings indicated that the performance of TD adolescents, but not that of participants with ASD, became worse in the inverted scene condition. This coincided with a drop in correlation strength between the participant performance in the upright and inversed condition of both the ASD and TD group and image difficulty / change predictability / 'clue' needed. Nonetheless, in the inverted scene condition, this correlation was found to be significantly stronger for the TD participants. These observations were in line with previous findings in TD participants arguing that scene inversion has a disruptive effect on the global gist perception and semantic evaluations of meaningful scenes (Epstein et al., 2006; Kelley et al., 2003; Shore and Klein, 2000). This preattentive and automatic global processing in TD participants, to get a first rapid awareness of the conceptual gist of the scene, is generally followed by a more conscious focus of attention to specific low-level elements in the display (Hochstein and Ahissar, 2002; Ahissar and Hochstein, 2004). When this initial gist perception is less efficient, the later detail-specific search strategy might also be delayed. This seemed less the case in adolescents with ASD, who have a stronger 
inclination or preference to process local stimulus elements than TD people (Happé and Booth, 2008; Mottron et al., 2006).

Such a weaker emphasis on rapidly extracting the global stimulus properties coinciding with a hyper-activation of detailed, but low-level, scene elements in ASD, could explain the superior perceptual discrimination and diminished processing of common features, for instance, during feature detection in inverted scenes (Plaisted, 2001). More precisely, when participants are required to consciously focus attention on local scene (object) information within an incoherent (or inverted) whole, people with ASD generally show an enhanced ability, compared to TD participants, to discriminate between locally defined stimuli embedded within this larger context (Van der Hallen et al., 2015; Vanmarcke et al., 2016). As the a posteriori correlation analysis indicated, this might result from a stronger shift to a more locally biased search strategy in people with ASD, compared to TD participants, in tasks in which the rapid processing of global information does not help to improve change detection performance (Fletcher-Watson et al., 2012; Van der Hallen et al., 2015). Therefore, it might be that the default mode of processing in TD perceivers is always to extract the gist first in meaningful visual displays, even when this gist is not readily interpretable (e.g., inverted scenes) and task performance improves by quickly focusing on detailed scene information. Nonetheless, when our task would specifically focus on rapidly extracting global meaning, people with ASD are expected to become less efficient than TD 
participants (e.g., when changes involve highly meaningful scene elements). To better understand these findings, we then tested whether change location and size directly (or indirectly) mediated these group-level differences in change detection performance.

Change location. All participants, without group-level differences, were better in detecting changes further away from the image center than in detecting those near to the natural focus of attention. This was similar to the findings of Utochkin (2011) in TD adults, implying that the natural focus of attention in the current database coincided with the physical center of the images due to the fixation cross preceding each experimental trial. Nonetheless, our results were in contradiction with the study of Sareen et al. (2015), using the same images as in the current study, in which no effect of change location was found. This might be explained by the methodological differences between their and our experimental set-up. More precisely, in their study (1) only a limited, adult, participant sample was tested, (2) participants provided a go-/no-go response when the change was detected (no 2-AFC task design) and (3) no scene inversion was implemented during testing. Interestingly, our measure of semantic centrality indicated that most changes in the current database were regarded as less semantically central. As a result, we believe that follow-up research will need to focus on enlarging the variability of both (1) the semantic meaningfulness of the changes and (2) the image context (e.g., outdoor scenes, social information,...). This will allow us to link the current findings, on the physical location of the changes within a scene, with 
previous observations on the role of semantic centrality on change detection performance in participants with and without ASD (Rensink et al., 1997; Smith and Milne, 2009).

Change size. All participants, without group-level differences, were faster to observe larger, compared to smaller, changes in the images. This was in line with our research hypothesis (Henderson and Hollingworth, 1999), but in contradiction to the study of Sareen and colleagues (2015). The latter might be explained, as with the findings on change location, by the methodological differences between their and our experimental set-up. Interestingly, and in contradiction with our expectations, we also did not observe group-level differences in size perception in the inverted scene condition. Simultaneously, we also did not observe meaningful a posteriori correlations between participant performance and non-semantic image properties (visual saliency, self-similarity, complexity and anisotropy) in this condition. Nonetheless, the perception of size might interact with other perceptual (e.g., color) and semantic (e.g., scene congruency) image properties and thereby influencing change blindness detection indirectly (Stirk and Underwood, 2007). This is in line with studies suggesting that a model of eye guidance, only based on the (low-level) visual properties of a scene, cannot fully account for adequate scene perception during change detection (Wright, 2005). More probable are models of eye guidance that allow for rapid global top-down information processing to bias the allocation of attention within a scene, 
leading to a faster or slower detection of visual changes (Ahissar and Hochstein, 2004; Torralba, 2003).

Age as a predictor of change detection performance. Finally, we found a significant influence of age on participant performance, indicating that older adolescents were better in detecting changes than their younger counterparts (similar to Shore et al., 2006). This was in line with previous research indicating that semantic categorization of visual information undergoes a specific developmental trajectory throughout childhood (Batty and Taylor, 2002; Fletcher-Watson et al., 2009a), coinciding with a steadily increasing response speed (Kail, 1993) and an immaturity in sustained attention and inhibition in adolescence (Jonkman et al., 2003). Interestingly, in our study, adolescents with and without ASD showed a similar age-related improvement in change detection performance, with an overall better performance of people with ASD (compared to their TD counterparts) in the inverted scene condition. Previous research with younger children (ranging from 4 to 13 years old) did not observe an enhanced detection performance in ASD (Burack et al., 2009). However, these authors only reported developmental improvement of change detection in TD children, not in children with ASD. It was thereby suggested that change detection is characterized by an atypical developmental trajectory in ASD, related to overall differences in (local/global) attentional search strategy (following from, e.g., enhanced discrimination abilities in ASD). This idea is in line with change blindness studies using complex 
visual displays, instead of pairs of images of isolated objects (as used by Burack et al., 2009), reporting that adolescents with ASD outperformed their TD peers during change detection (Fletcher-Watson et al., 2012; Smith and Milne, 2009). This could either suggest that enhanced attention in ASD may only become apparent when displaying changes to items within complex scenes or that differences in change detection between people with and without ASD critically arise in the transition from childhood to adolescence.

Study limitation. In order to avoid showing the same images twice to the participants, we choose to conduct the stimuli ratings independently from the main experiment. However, we were unable to test a second (independent) sample of participants with ASD and could therefore only administer the stimulus rating task to an independent sample of TD participants. This was not considered to be a major problem, given that the main interest of the rating task was to test whether there were group-level differences in change detection strategy. The ratings of the TD group could therefore function as a baseline measure, which was strongly correlated with the performance of the TD adolescents and was then further compared with the performance of the adolescents with ASD (to test for possible group-level differences). Nonetheless, we cannot exclude the possibility that the lower correlation between the TD ratings and the change blindness performance of the participants with ASD followed from a different notion of meaningfulness and/or predictability in both participant groups. This 
alternative explanation should be addressed in future research using a similar change detection paradigm.

Future research. The current change blindness study used the image database by Sareen and colleagues (2015), which only contains complex, non-social scenes. We believe that future research should also incorporate socially-relevant stimuli (and changes). This is especially relevant for better understanding the differences in social attention between people with and without ASD, which generally remain subtle in studies using a free description task to evoke an open assessment of social interest in a naturalistic visual scene (Fletcher-Watson et al., 2013; Freeth et al., 2011; Vanmarcke et al., 2016). Furthermore, these differences in social attention often only become more prominent in automatic, implicit measurements such as viewing preference based on the location of the first fixation (Fletcher-Watson et al., 2009). The latter finding could be related to the idea of a reduced salience of social stimuli and concomitant enactment of socially irrelevant aspects of the environment (Klin et al., 2003). It would be interesting to see whether similar observations are possible within a change blindness experiment, using both upright and inverted scene conditions.

\section{Conclusion}


All participants, with and without ASD, performed similar when scenes were presented upright. When the images were inverted, only the performance of the TD adolescents became worse. This might follow from a stronger shift to a more locally biased search strategy in people with ASD, compared to TD participants, in tasks in which the rapid processing of global information does not help to improve change detection performance. Nonetheless, when our task would specifically focus on extracting global meaning, people with ASD are expected to become less efficient than TD participants. Importantly, although we found that change location, change size and age also influenced participant performance, they could not be linked to the performance differences between adolescents with and without ASD in the inversed scene condition. We therefore believe that follow-up research will need to focus on (1) enlarging the image variability (semantic meaningfulness, image context,...) and (2) systematically measuring/quantifying those scene characteristics correlated with participant performance (predictability, task difficulty, change location/size,...). 
Ahissar M, Hochstein S (2004) The reverse hierarchy theory of visual perceptual learning. Trends in Cognitive Sciences 8: 457-464.

American Psychiatric Association. (2000). Diagnostic and statistical manual of mental disorders (4th ed., text revision). American Psychiatric Publishing, Inc.

American Psychiatric Association (2013) Diagnostic and statistical manual of mental disorders (5th ed.). American Psychiatric Publishing, Inc.

Ames C, Fletcher-Watson S (2010) A review of methods in the study of attention in autism. Developmental Review 30: 52-73.

Ariely D (2001) Seeing sets: Representation by statistical properties. Psychological Science 12: 157-162.

Batty M, Taylor MJ (2002) Visual categorization during childhood: an ERP study. Psychophysiology 39: 482-490.

Braun J, Amirshahi S, Denzler J, et al. (2013) Statistical image properties of print advertisements, visual artworks and images of architecture. Frontiers in Psychology 4: 1-15. doi: 10.3389/fpsyg.2013.00808.

Bullier J (2001) Integrated model of visual processing. Brain Research Reviews 36: 96107. 
Burack JA, Joseph S, Russo N, et al. (2009). Change detection in naturalistic pictures among children with autism. Journal of Autism and Developmental Disorders 39: 471-479.

Dakin S, Frith U (2005) Vagaries of visual perception in autism. Neuron 48: 497507.

de Bildt A, Sytema S, van Lang NDJ, et al. (2009) Evaluation of the ADOS Revised Algorithm: The Applicability in 558 Dutch Children and Adolescents. Journal of Autism and Developmental Disorders 39: 1350-1358. http://doi.org/10.1007/s10803-009-0749-9

Epstein RA, Higgins JS, Parker W, et al. (2006). Cortical correlates of face and scene inversion: a comparison. Neuropsychologia 44: 1145-1158.

Fletcher-Watson S, Collis JM, Findlay JM, et al. (2009a) The development of change blindness: children's attentional priorities whilst viewing naturalistic scenes. Developmental Science 12: 438-445.

Fletcher-Watson S, Leekam SR, Benson V, et al. (2009b) Eye-movements reveal attention to social information in autism spectrum disorder. Neuropsychologia 47: $248-257$.

Fletcher-Watson S, Leekam SR, Connolly B, et al. (2012) Attenuation of change blindness in children with autism spectrum disorders. British Journal of Developmental Psychology 30: 446-458. 
Fletcher-Watson S, Leekam SR, and Findlay, JM (2013) Social interest in high functioning adults with autism spectrum disorders. Focus on Autism and Other Developmental Disabilities 28: 222-229.

Fletcher-Watson S, Leekam SR, Turner MA, et al. (2006) Do people with autistic spectrum disorder show normal selection for attention? Evidence from change blindness. British Journal of Psychology 97: 537-554.

Freeth M, Ropar D, Mitchell P, et al. (2011) Brief report: how adolescents with ASD process social information in complex scenes. Combining evidence from eye movements and verbal descriptions. Journal of Autism and Developmental Disorders 41: 364-371.

Gotham K, Risi S, Pickles A, et al. (2006) The Autism Diagnostic Observation Schedule: Revised Algorithms for Improved Diagnostic Validity. Journal of Autism and Developmental Disorders 37: 613-627. doi: http://doi.org/10.1007/s 10803-006-0280-1

Happé F, Booth R (2008) The power of the positive: Revisiting weak coherence in autism spectrum disorders. The Quarterly Journal of Experimental Psychology 61: $50-63$.

Henderson JM, Hollingworth A (1999) The role of fixation position in detecting scene changes across saccades. Psychological Science 10: 438-443. 
Hochstein S, Ahissar M (2002) View from the top: Hierarchies and reverse hierarchies in the visual system. Neuron 36: 791-804.

Jonkman LM, Lansbergen M, and Stauder, JEA (2003) Developmental differences in behavioral and event-related brain responses associated with response preparation and inhibition in a go/nogo task. Psychophysiology 40: 752-761.

Kail R (1993) Processing time decreases globally at an exponential rate during childhood and adolescence. Journal of Experimental Child Psychology 56: 254265.

Kaiser MD, Shiffrar M (2009) The visual perception of motion by observers with autism spectrum disorders: A review and synthesis. Psychonomic Bulletin \& Review 16: 761-777.

Kauffmann L, Ramanoël S, and Peyrin C (2014) The neural bases of spatial frequency processing during scene perception. Frontiers in Integrative Neuroscience 8: 114. doi: 10.3389/fnint.2014.00037.

Kelley TA, Chun MM, and Chua, KP (2003) Effects of scene inversion on change detection of targets matched for visual salience. Journal of Vision 3: 1-5. doi: 10.1167/3.1.1.

Kikuchi Y, Senju A, Tojo Y, et al. (2009) Faces do not capture special attention in children with autism spectrum disorder: A change blindness study. Child Development 80: 1421-1433. 
Klin A, Jones W, Schultz R, et al. (2003) The enactive mind, or from actions to cognition: lessons from autism. Philosophical Transactions of the Royal Society B: Biological Sciences 358: 345-360.

Koldewyn K, Jiang YV, Weigelt S, et al. (2013) Global/local processing in autism: Not a disability, but a disinclination. Journal of Autism and Developmental Disorders 43: 2329-2340.

Landman R, Spekreijse H, and Lamme VA (2003). Large capacity storage of integrated objects before change blindness. Vision Research 43: 149-164.

Loth E, Gómez JC, and Happé F (2008) Event schemas in autism spectrum disorders: The role of theory of mind and weak central coherence. Journal of Autism and Developmental Disorders 38: 449-463.

Maccari L, Pasini, A, Caroli E, et al. (2014). Visual Search and Emotion: How Children with Autism Spectrum Disorders Scan Emotional Scenes. Journal of Autism and Developmental Disorders 44: 2871-2881.

McArdle BH (1987) The significance of differences between means. A simulation study. Comparative Biochemistry and Physiology 87A: 979-982.

McCullagh P (1984) Generalized linear models. European Journal of Operational Research 6: 285-292. 
Mottron L, Dawson M, Soulières I, et al. (2006) Enhanced perceptual functioning in autism: An update, and eight principles of autistic perception. Journal of Autism and Developmental Disorders 36: 27-43.

O'Regan J, Deubel H, Clark JJ, et al. (2000) Picture changes during blinks: Looking without seeing and seeing without looking. Visual Cognition 7: 191-211.

Plaisted KC (2001) Reduced generalization in autism: An alternative to weak central coherence. The development of autism: Perspectives from Theory and Research 2: $149-169$.

Rakover SS, Cahlon, B (2001) Face recognition: Cognitive and computational processes (Vol. 31). Amsterdam/Philadelphia: John Benjamins Publishing Company.

$\mathrm{R}$ Core Team (2013) R: A language and environment for statistical computing. $\mathrm{R}$ Foundation for Statistical Computing, Vienna, Austria. ISBN 3-900051-07-0.

Rensink RA, O'Regan JK, and Clark JJ (1997) To see or not to see: The need for attention to perceive changes in scenes. Psychological Science 8: 368-373.

Roeyers H, Thys M, Druart C, et al. (2011) SRS: screeningslijst voor autismespectrumstoornissen, handleiding. Hogrefe Uitgevers, Amsterdam.

Sareen P, Ehinger KA, and Wolfe JM (2015) CB Database: A change blindness database for objects in natural indoor scenes. Behavior research methods, 1-6. 
Sattler JM (2001) Assessment of children: Cognitive applications (4th ed.). San Diego, CA: Jerome M Sattler Publisher Inc. 931 p.

Schyns PG, Oliva A (1994) From blobs to boundary edges: Evidence for time- and spatial-scale-dependent scene recognition. Psychological Science 5: 195-200.

Senju A (2013) Atypical development of spontaneous social cognition in autism spectrum disorders. Brain and Development 35: 96-101.

Shore DI, Burack JA, Miller D, et al. (2006) The development of change detection. Developmental Science 9: 490-497. doi: 10.1111/j.1467-7687.2006.00516.

Shore DI, Klein, RM (2000) The effects of scene inversion on change blindness. The Journal of General Psychology 127: 27-43.

Simons DJ, Rensink RA (2005). Change blindness: Past, present, and future. Trends in Cognitive Sciences 9: 16-20.

Simons DJ, Ambinder, MS (2005) Change blindness theory and consequences. Current Directions in Psychological Science 14: 44-48.

Smith H, Milne E (2009) Reduced change blindness suggests enhanced attention to detail in individuals with autism. Journal of Child Psychology and Psychiatry 50: $300-306$.

Steiger JH (1980) Tests for comparing elements of a correlation matrix. Psychological Bulletin 87: 245-251. 
Stirk JA, Underwood G (2007) Low-level visual saliency does not predict change detection in natural scenes. Journal of Vision 7: 1-10. doi: 10.1167/7.10.3.

Torralba A (2003) Contextual priming for object detection. International Journal of Computer Vision 53: 169-191.

Utochkin IS (2011) Hide-and-seek around the centre of interest: The dead zone of attention revealed by change blindness. Visual Cognition 19: 1063-1088.

Van Belle G, De Graef P, Verfaillie K, et al. (2010) Face inversion impairs holistic perception: Evidence from gaze-contingent stimulation. Journal of Vision 10: 113. doi:10.1167/10.5.10.

Van der Hallen R, Evers K, Brewaeys K, et al. (2015) Global processing takes time: A meta-analysis on local-global visual processing in ASD. Psychological Bulletin 141: 549-573. doi: http://dx.doi.org/10.1037/bul0000004.

Vanmarcke S, Mullin C, Van der Hallen R, et al. (2016) In the eye of the beholder: Rapid visual perception of real-life scenes by young adults with and without ASD. Journal of Autism and Developmental Disorders 46: 2635-2652. doi: 10.1007/s10803-016-2802-9.

Wald A (1943) Tests of statistical hypotheses concerning several parameters when the number of observations is large. Transactions of the American Mathematical Society 54: 426-482. 
Walther D, Koch, C (2006) Modeling attention to salient proto-objects. Neural Networks 19: 1395-1407.

Wang S, Jiang M, Duchesne XM, et al. (2015) Atypical visual saliency in autism spectrum disorder quantified through model-based eye tracking. Neuron 88 : 604-616.

Wechsler D (1997) Wechsler adult intelligence scale. (3rd edition). San Antonio, The Psychological Corporation.

Wright MJ (2005) Saliency predicts change detection in pictures of natural scenes. Spatial Vision 18: 413-430. 


\section{Figure captions}

Figure 1. In (A) a graphical overview of the trial design of the change blindness task is provided. Participants were presented with an image $(240 \mathrm{~ms})$, followed by a blank (grey background; $240 \mathrm{~ms})$, an altered image (240 ms) and a blank (240 ms). Afterwards the process repeated itself until the participant pressed a response. In (B), some example images are shown. The orange blob on the grey square thereby exemplifies the differences in change location and size in the different images. The picture set is available on http://search.bwh.harvard.edu/new/CBDatabase.html.

Figure 2. Overview of the RT data in the change blindness task. In (A), the data are represented as the mean performance across participants, with error bars depicting the standard error of the mean (SEM). In (C), the data are represented as the individual performance of each participant in a scatter plot, with the upright images on the abscissa and the inverted images on the ordinate. In all panels, adolescents with ASD were depicted in blue and TD adolescents in red.

Figure 3. Visualization of the data by means of scatterplots. The mean RT per image was indicated on the ordinate axis, while in (A) we depicted the image difficulty (in mean RT), in (B) the rated semantic centrality, in (C) the rated predictability and in (D) 
the proportion of people requesting a 'clue' to find the change in the rating task on the abscissa. In all panels, the performance of adolescents with ASD in the upright condition was depicted in green and their performance in the inverted condition in blue. Simultaneously, the performance of the TD adolescents in the upright condition was depicted in purple and their performance in the inverted condition in dark grey. 
Figure 1

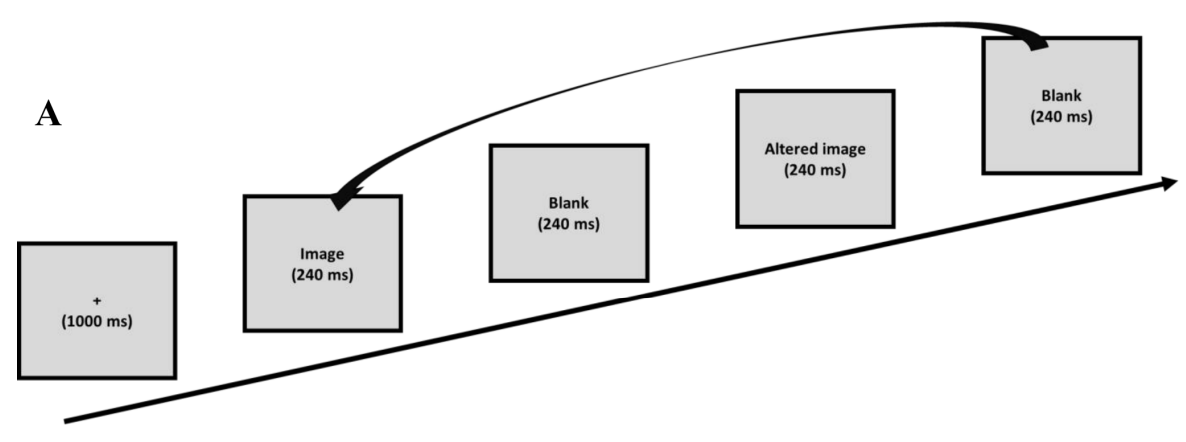

B
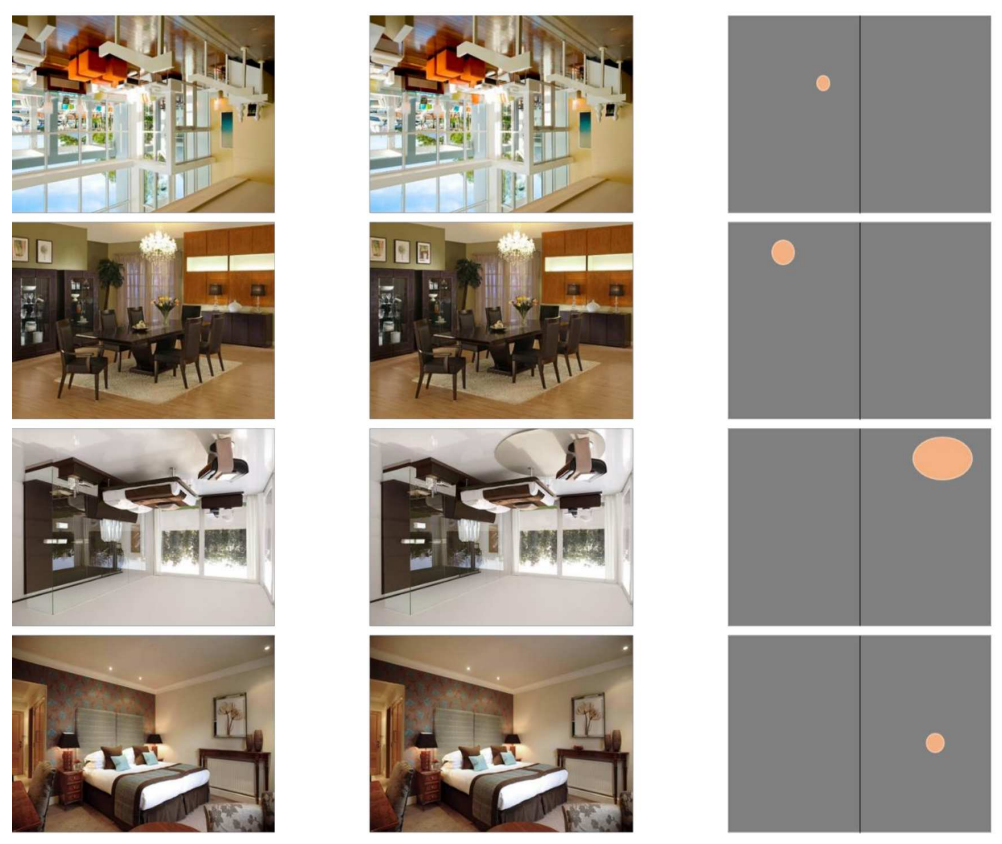

47

48

49

50 
Figure 2
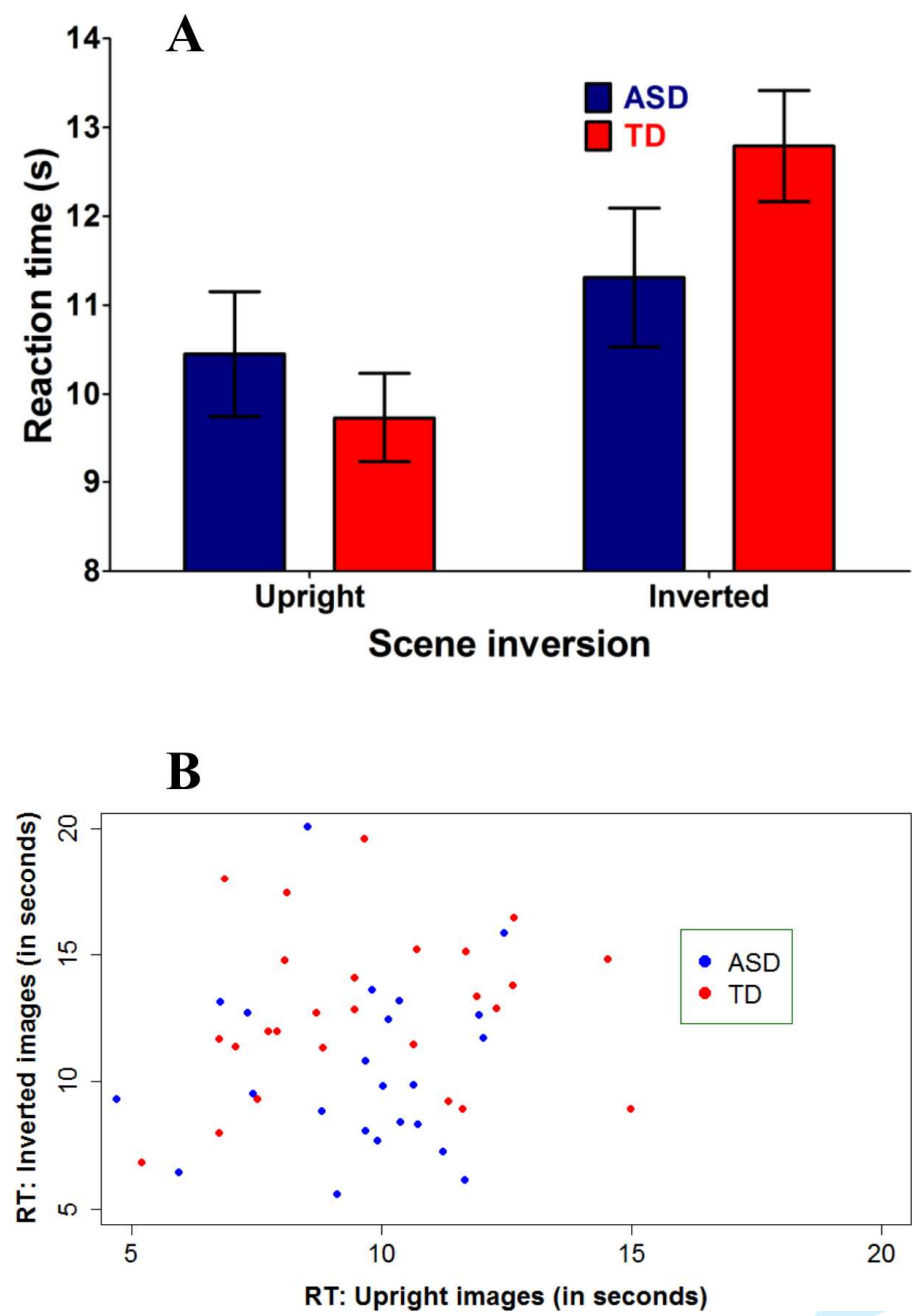


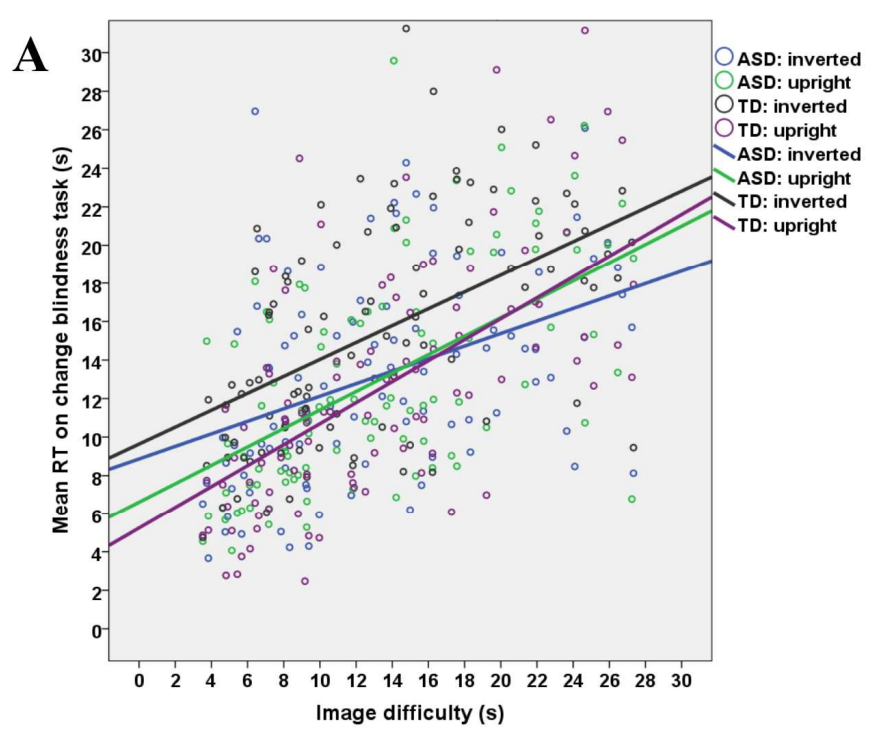

Figure 3
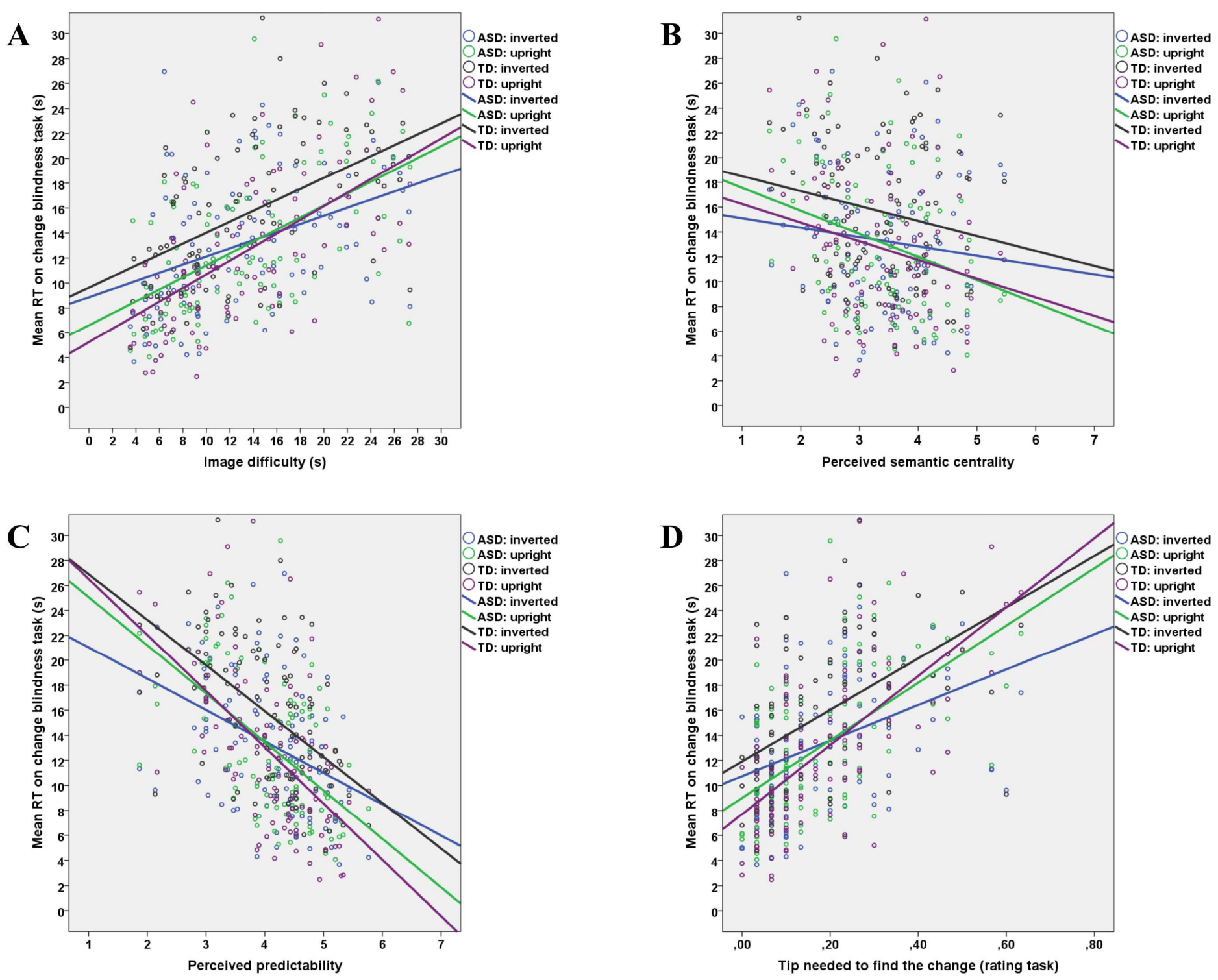
Table 1. Overview of the average group-level performance (SD between brackets), for participants with ASD $(n=26)$ and TD participants $(n=26)$. On each of the descriptive tests separately, an independent-samples t-test with Group (TD versus ASD) as between-participants factor was conducted. 
1

2

3

4

5

6

7

8

9

10

Table 2. Overview of the correlation matrix between the mean RT performance per image, for the group-level specific performance (TD versus ASD) in the upright and inverted condition separately, and (1) image difficulty, (2) horizontal and vertical pixel location, (3) the visual saliency of the change, (4) the rated predictability and (5) semantic centrality of the changes, (6) whether or not a clue was needed to detect the changes during the rating task and (7) measures of non-semantic image properties such as self-similarity, complexity and anisotropy.

\begin{tabular}{|c|c|c|c|c|c|c|c|c|c|c|}
\hline & $\begin{array}{l}\text { Image } \\
\text { difficulty }\end{array}$ & $\begin{array}{c}\text { Horizontal } \\
\text { change } \\
\text { location }\end{array}$ & $\begin{array}{l}\text { Vertical } \\
\text { change } \\
\text { location }\end{array}$ & $\begin{array}{c}\text { Visual } \\
\text { saliency }\end{array}$ & Predictability & $\begin{array}{l}\text { Semantic } \\
\text { centrality }\end{array}$ & $\begin{array}{l}\text { Clue } \\
\text { needed }\end{array}$ & $\begin{array}{l}\text { Image self- } \\
\text { similarity }\end{array}$ & $\begin{array}{c}\text { Image } \\
\text { complexity }\end{array}$ & $\begin{array}{c}\text { Image } \\
\text { anisotropy }\end{array}$ \\
\hline TD (upright scenes) & $.66^{*}$ & -.11 & .03 & .02 & $-.59 *$ & -.21 & $.65^{*}$ & .16 & .23 & -.12 \\
\hline TD (inverted scenes) & $.55^{*}$ & -.08 & .11 & .02 & $-.50 *$ & -.18 & $.50 *$ & .18 & .05 & -.04 \\
\hline ASD (upright scenes) & $.59^{*}$ & .14 & -.08 & .07 & $-.55^{*}$ & -.28 & $.58^{*}$ & .09 & .06 & -.01 \\
\hline ASD (inverted scenes) & $.41^{*}$ & -.06 & .17 & -.10 & $-.38 *$ & -.12 & $.38 *$ & .01 & .02 & -.08 \\
\hline
\end{tabular}

(* Bonferroni corrected level: $p<.001)$ 


\section{Supplementary materials}

\section{Perceived predictability, semantic centrality and 'clue' needed}

We let an independent group of TD participants $(n=30)$, with a mean age of $36.67(S D=16.61)$, rate the predictability (How predictable is the change in the scene?) and the semantic centrality (How meaningful is the change in the scene?) of each of the changes, in each of the images, on a 7-point Likert scale (Figure 1Sup). Lower values thereby indicated less and higher values more perceived predictability/semantic centrality of the change. To complete this task, both the original and the altered version of each image were shown simultaneously, and without time constraints, to the participants. If they were not able to locate the change in the scene themselves, they were allowed to ask for a 'clue'. This 'clue' unambiguously illustrated the exact location of the change Overall, the mean perceived predictability was $4.08(S D=.82)$, the mean perceived semantic centrality was $3.37(S D=.88)$ and the mean proportion of participants needing a clue to locate a change in the image was $.18(S D=.15)$. In order to check the internal consistency of the measured variables, we randomly split our group of 30 participants in half, providing us with two groups of 15 participants, with a mean age of $36.33(S D=15.21)$ in Group 1 and a mean age of $37.00(S D=18.45)$ in Group 2. Correlating the mean scores on perceived predictability, semantic centrality and 'clue' between both groups thereby clearly indicated that there was a strong interrater-agreement in image scores (Table 1Sup). As a results, we correlated the mean scores per image, for all three variables, in the total participant set of 30 participants with the mean RT scores per image, for the group-level specific performance (ASD and TD) in the upright and inverted condition separately, of the change blindness task. 

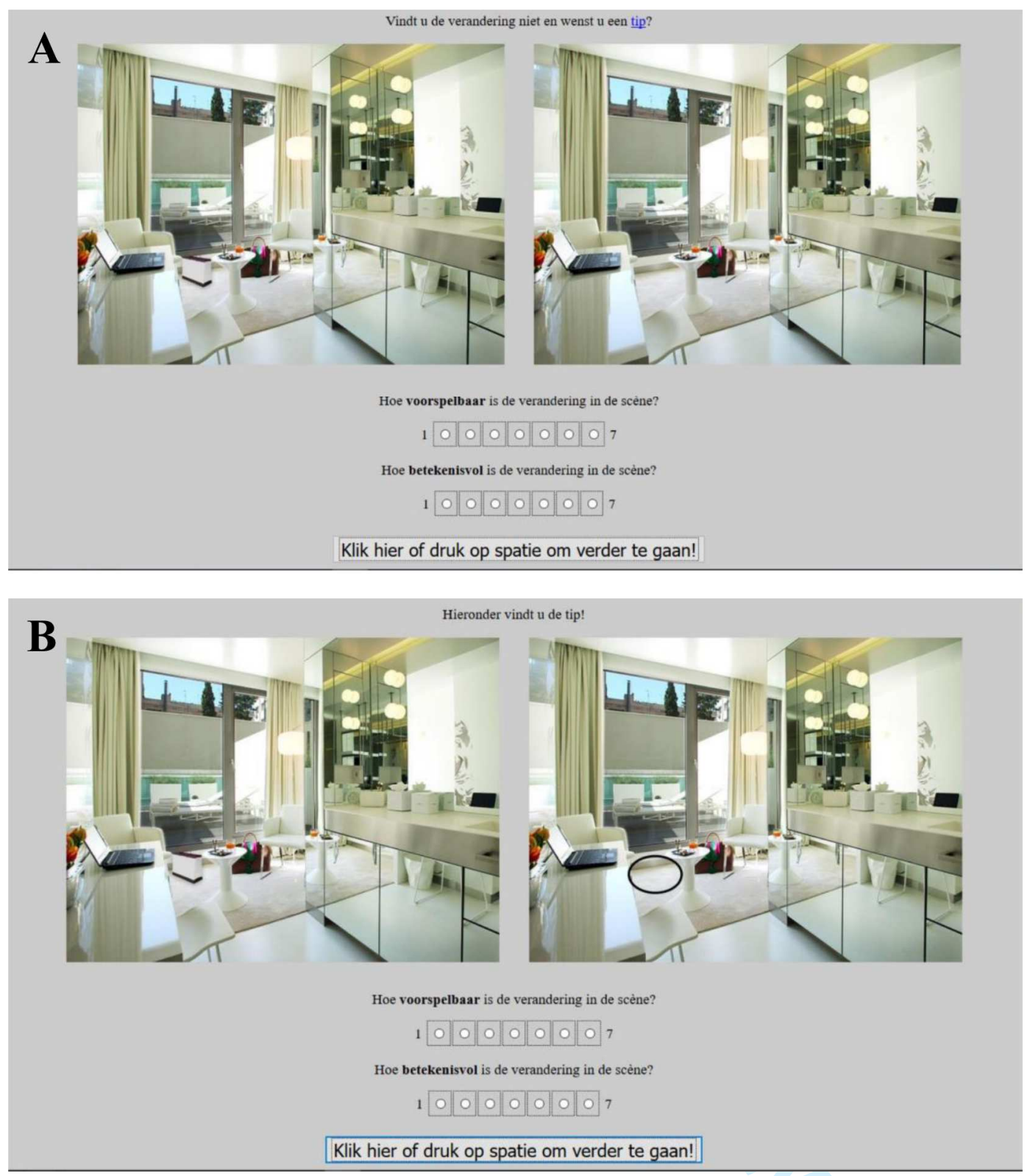

Figure 1Sup. Graphical example of the rating task, conducted by 30 TD participants. In (A), the original and altered version of an image are shown, together with both questions (in Dutch) asked to our participants: (1) How predictable is the change in the scene? and (2) How meaningful is the change in the scene?. In (B), the participant asked for the 'clue', which is then presented at the location of the unaltered image. In this image, the black circle unambiguously illustrated the exact location of the change. 
Table 1Sup. Overview of the correlation matrix between the image ratings of Group $1(n=15)$ and Group $2(n=15)$. This was done for the following three variables: (1) perceived predictability, (2) perceived semantic centrality and (3) 'clue' needed.

\begin{tabular}{cccc}
\hline & $\begin{array}{c}\text { Perceived predictability } \\
\text { (Group 1) }\end{array}$ & $\begin{array}{c}\text { Perceived semantic centrality } \\
\text { (Group 1) }\end{array}$ & $\begin{array}{c}\text { Clue needed } \\
\text { (Group 1) }\end{array}$ \\
$\begin{array}{c}\text { Perceived predictability } \\
\text { (Group 2) }\end{array}$ & $\mathbf{. 8 6}^{*}$ & $.56^{*}$ & $-.71^{*}$ \\
$\begin{array}{c}\text { Perceived semantic centrality } \\
\text { (Group 2) } \\
\begin{array}{c}\text { Clue needed } \\
\text { (Group 2) }\end{array}\end{array}$ & $.38^{*}$ & $\mathbf{. 8 9 *}$ & -.24 \\
\hline
\end{tabular}

$(*$ Bonferonni corrected level: $p<.006)$ 


\title{
Results of the GLMM model selection process for the change blindness task, in terms of fixed effects and goodness-of-fit, for both RT and accuracy.
}

\begin{abstract}
Overview of the parameter estimates for the change detection task for both (a) the random intercepts regression analysis on the RT output and (b) the random intercepts logistic regression analysis on the accuracy data.
\end{abstract}

\begin{tabular}{|c|c|c|c|}
\hline \multicolumn{4}{|c|}{ RT } \\
\hline Parameter & Estimate (Standard Error) & p-value & $95 \%$ confidence interval \\
\hline Intercept & $24.26(3.05)$ & $<.001$ & {$[18.28 ; 30.24]$} \\
\hline Group & $-.26(.71)$ & .71 & {$[-1.65 ; 1.13]$} \\
\hline Age & $-.60(.21)$ & $<.01$ & {$[-1.01 ;-.19]$} \\
\hline Scene inversion & $.30(.55)$ & .59 & {$[-.78 ; 1.38]$} \\
\hline Change location & $-5.60 * 10^{-3}\left(1.54 * 10^{-3}\right)$ & $<.001$ & {$\left[-8.62 * 10^{-3} ;-2.58 * 10^{-3}\right]$} \\
\hline Change size & $-1.06 * 10^{-4}\left(3.28 * 10^{-5}\right)$ & $<.01$ & {$\left[-1.70 * 10^{-4} ;-4.17 * 10^{-5}\right]$} \\
\hline Image order & $-.05(.01)$ & $<.001$ & {$[-.07 ;-.03]$} \\
\hline Group * Scene inversion & $2.64(.77)$ & $<.001$ & {$[1.13 ; 4.15]$} \\
\hline \multicolumn{4}{|c|}{ Accuracy } \\
\hline Parameter & Estimate (Standard Error) & p-value & $95 \%$ confidence interval \\
\hline Intercept & $-2.91(.94)$ & $<.01$ & {$[-4.75 ;-1.07]$} \\
\hline Group & $.12(.23)$ & .60 & {$[-.33 ; .57]$} \\
\hline Age & $.18(.06)$ & $<.01$ & {$[.06 ; .30]$} \\
\hline Scene inversion & $-.35(.19)$ & .07 & {$[-.72 ; .02]$} \\
\hline Change location & $3.25 * 10^{-3}\left(5.58 * 10^{-4}\right)$ & $<.001$ & {$\left[2.16 * 10^{-3} ; 4.34 * 10^{-3}\right]$} \\
\hline Change size & $-1.30 * 10^{-5}\left(1.14 * 10^{-5}\right)$ & .25 & {$\left[-3.53 * 10^{-5} ;-9.34 * 10^{-6}\right]$} \\
\hline Image order & $9.03 * 10^{-3}\left(4.60 * 10^{-3}\right)$ & .05 & {$\left[1.40 * 10^{-5} ; .02\right]$} \\
\hline Group * Scene inversion & $.12(.27)$ & .67 & {$[-.41 ; .65]$} \\
\hline
\end{tabular}




\section{A. Trial-by-trial reaction time}

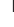

2

3

5

9

Fixed effects Model A Model B Model C

44 Change location * Change

\section{5}

\section{4}

4

4

4

50

51

53

54

55 


7

8

8

9

10

\section{4}

4

4

\section{B. Trial-by-trial accuracy}

\begin{tabular}{|c|c|c|c|c|c|c|c|c|}
\hline Fixed effects & Model A & Model B & Model C & Model D & Model E & Model F & Model G & Model H \\
\hline Intercept & $\begin{array}{l}.60^{* * *} \\
(.10)\end{array}$ & $\begin{array}{l}.45 * * \\
(.14)\end{array}$ & $\begin{array}{l}.72 * * * \\
(.20)\end{array}$ & $\begin{array}{c}-1.79 * 10^{-3} \\
(.36)\end{array}$ & $\begin{array}{l}.05 \\
(.36)\end{array}$ & $\begin{array}{l}-4.34 * * \\
(1.64)\end{array}$ & $\begin{array}{l}-4.14 * * \\
(1.64)\end{array}$ & $\begin{array}{c}-2.91 * * \\
(.94)\end{array}$ \\
\hline Group & --- & $\begin{array}{l}.29 \\
(.19)\end{array}$ & $\begin{array}{l}.20 \\
(.28)\end{array}$ & $\begin{array}{l}.05 \\
(.43)\end{array}$ & $\begin{array}{l}-.01 \\
(.44)\end{array}$ & $\begin{array}{l}2.96 \\
(2.04)\end{array}$ & $\begin{array}{l}3.04 \\
(2.15)\end{array}$ & $\begin{array}{l}.12 \\
(.23)\end{array}$ \\
\hline Scene inversion & --- & -- & $\begin{array}{l}-.43^{*} \\
(.21)\end{array}$ & $\begin{array}{l}-.63 \\
(.41)\end{array}$ & $\begin{array}{l}-.63 \\
(.41)\end{array}$ & $\begin{array}{l}-.63 \\
(.47)\end{array}$ & $\begin{array}{l}-.63 \\
(.41)\end{array}$ & $\begin{array}{l}-.35 \\
(.19)\end{array}$ \\
\hline Change location & --- & --- & -- & $\begin{array}{l}2.39 * 10^{-3 *} \\
\left(1.00 * 10^{-3}\right)\end{array}$ & $\begin{array}{l}2.56 * 10^{-3 * *} \\
\left(9.84 * 10^{-4}\right)\end{array}$ & $\begin{array}{l}2.80 * 10^{-3 * *} \\
\left(9.83 * 10^{-4}\right)\end{array}$ & $\begin{array}{l}2.80 * 10^{-3 * *} \\
\left(9.84 * 10^{-4}\right)\end{array}$ & $\begin{array}{c}3.25 * 10^{-3 * * *} \\
\left(5.58 * 10^{-4}\right)\end{array}$ \\
\hline Change size & --- & -- & -- & -- & $\begin{array}{l}-2.45 * 10^{-5} \\
\left(1.33 * 10^{-5}\right)\end{array}$ & $\begin{array}{l}-2.58 * 10^{-5} \\
\left(1.83 * 10^{-5}\right)\end{array}$ & $\begin{array}{l}-2.57 * 10^{-5} \\
\left(1.82 * 10^{-5}\right)\end{array}$ & $\begin{array}{l}-1.30 * 10^{-5} \\
\left(1.14 * 10^{-5}\right)\end{array}$ \\
\hline Correct response (left/right) & --- & -- & --- & --- & --- & $\begin{array}{l}.10 \\
(.14)\end{array}$ & $\begin{array}{l}.09 \\
(.14)\end{array}$ & --- \\
\hline FSIQ & --- & -- & -- & --- & --- & $\begin{array}{l}.01 \\
(.01)\end{array}$ & $\begin{array}{l}.01 \\
(.01)\end{array}$ & --- \\
\hline Age & --- & --- & -- & --- & --- & $\begin{array}{l}.21 * * \\
(.07)\end{array}$ & $\begin{array}{l}.21 * * \\
(.07)\end{array}$ & $\begin{array}{l}.18^{* *} \\
(.06)\end{array}$ \\
\hline Gender & --- & -- & --- & --- & --- & $\begin{array}{c}3.41 \\
(2.00)\end{array}$ & $\begin{array}{c}3.40 \\
(2.00)\end{array}$ & --- \\
\hline Image order & --- & -- & -- & --- & -- & -- & $\begin{array}{l}8.97 * 10^{-3} \\
\left(4.60 * 10^{-3}\right)\end{array}$ & $\begin{array}{l}9.03 * 10^{-3 *} \\
\left(4.60 * 10^{-3}\right)\end{array}$ \\
\hline Age * Gender & --- & --- & --- & --- & --- & $\begin{array}{l}-.21 \\
(.14)\end{array}$ & $\begin{array}{l}-.21 \\
(.14)\end{array}$ & --- \\
\hline Group * FSIQ & --- & --- & -- & --- & --- & $\begin{array}{l}-.03 \\
(.02)\end{array}$ & $\begin{array}{l}-.03 \\
(.02)\end{array}$ & --- \\
\hline Group * Scene inversion & --- & --- & $\begin{array}{l}.14 \\
(.30)\end{array}$ & $\begin{array}{l}.12 \\
(.30)\end{array}$ & $\begin{array}{l}.12 \\
(.31)\end{array}$ & $\begin{array}{l}.14 \\
(.31)\end{array}$ & $\begin{array}{l}.14 \\
(.31)\end{array}$ & $\begin{array}{l}.12 \\
(.27)\end{array}$ \\
\hline Group * Change location & --- & --- & -- & $\begin{array}{c}5.71 * 10^{-4} \\
\left(1.13 * 10^{-3}\right)\end{array}$ & $\begin{array}{c}3.90 * 10^{-4} \\
\left(1.13 * 10^{-3}\right)\end{array}$ & $\begin{array}{c}8.08 * 10^{-5} \\
\left(1.12 * 10^{-3}\right)\end{array}$ & $\begin{array}{l}8.08 * 10^{-5} \\
\left(1.12 * 10^{-3}\right)\end{array}$ & --- \\
\hline Group * Change size & --- & --- & -- & -- & $\begin{array}{c}2.81 * 10^{-5} \\
\left(1.91 * 10^{-5}\right)\end{array}$ & $\begin{array}{c}2.92 * 10^{-5} \\
\left(2.20 * 10^{-5}\right)\end{array}$ & $\begin{array}{l}2.92 * 10^{-5} \\
\left(2.20 * 10^{-5}\right)\end{array}$ & --- \\
\hline Group * Image order & -- & -- & --- & --- & --- & --- & $\begin{array}{l}-4.87 * 10^{-3} \\
\left(9.20 * 10^{-3}\right)\end{array}$ & --- \\
\hline $\begin{array}{l}\text { Scene inversion } * \text { Change } \\
\text { location }\end{array}$ & --- & --- & -- & $\begin{array}{l}7.46 * 10^{-4} \\
\left(1.16 * 10^{-3}\right)\end{array}$ & $\begin{array}{c}7.37 * 10^{-4} \\
\left(1.13 * 10^{-3}\right)\end{array}$ & $\begin{array}{c}7.14 * 10^{-4} \\
\left(1.13 * 10^{-3}\right)\end{array}$ & $\begin{array}{l}7.14 * 10^{-4} \\
\left(1.13 * 10^{-3}\right)\end{array}$ & --- \\
\hline $\begin{array}{l}\text { Scene inversion * Change } \\
\text { size }\end{array}$ & --- & --- & -- & -- & $\begin{array}{l}-1.06 * 10^{-6} \\
\left(1.76 * 10^{-5}\right)\end{array}$ & $\begin{array}{l}-8.13 * 10^{-7} \\
\left(2.21 * 10^{-5}\right)\end{array}$ & $\begin{array}{l}-8.13 * 10^{-7} \\
\left(2.21 * 10^{-5}\right)\end{array}$ & --- \\
\hline $\begin{array}{l}\text { Change location * Change } \\
\text { size }\end{array}$ & --- & --- & -- & --- & $\begin{array}{c}5.37 * 10^{-6} \\
\left(1.14 * 10^{-5}\right)\end{array}$ & $\begin{array}{c}5.37 * 10^{-6} \\
\left(1.14 * 10^{-5}\right)\end{array}$ & $\begin{array}{l}5.37 * 10^{-6} \\
\left(1.14 * 10^{-5}\right)\end{array}$ & --- \\
\hline
\end{tabular}

Goodness - of - fit

$\begin{array}{ccccccccc}\text { Deviance } & 2529.4 & 2527.1 & 2521.4 & 2489.4 & 2484.8 & 2471.2 & 2464.6 & 2480.6 \\ \text { Drop in Deviance } & --- & 2.3 & 5.7 & 32^{* * *} & 4.6 & 13.6^{*} & 6.6^{*} & -16 \\ \text { AIC } & 2537.4 & 2537.1 & 2535.4 & 2509.4 & 2510.8 & 2509.2 & 2506.6 & 2498.6 \\ \text { BIC } & 2561.0 & 2566.6 & 2576.7 & 2568.4 & 2587.6 & 2621.3 & 2626.6 & 2551.7\end{array}$


B
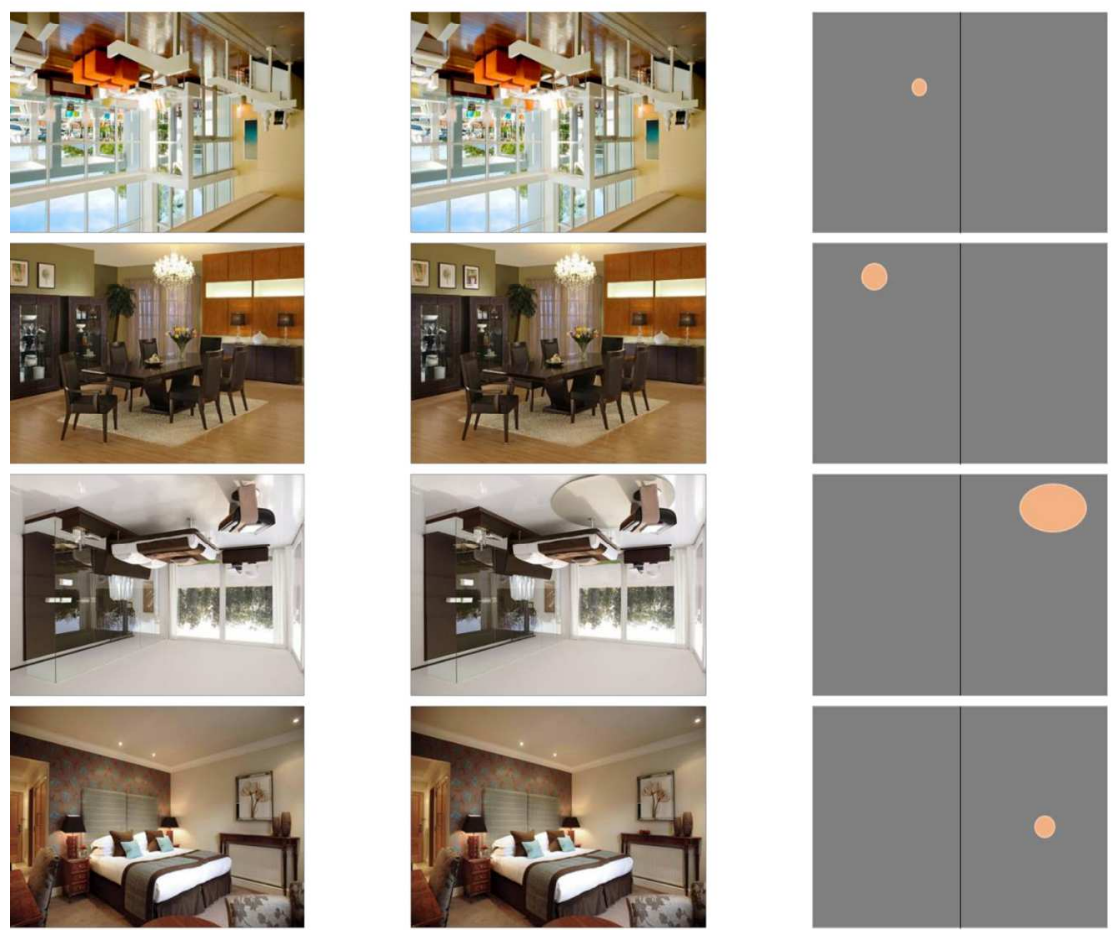

Figure 1. In (A) a graphical overview of the trial design of the change blindness task is provided. Participants were presented with an image $(240 \mathrm{~ms}$ ), followed by a blank (grey background; $240 \mathrm{~ms}$ ), an altered image $(240 \mathrm{~ms})$ and a blank $(240 \mathrm{~ms})$. Afterwards the process repeated itself until the participant pressed a response. In (B), some example images are shown. The orange blob on the grey square thereby exemplifies the differences in change location and size in the different images. The picture set is available on http://search.bwh.harvard.edu/new/CBDatabase.html.

$$
172 \times 206 \mathrm{~mm}(300 \times 300 \mathrm{DPI})
$$



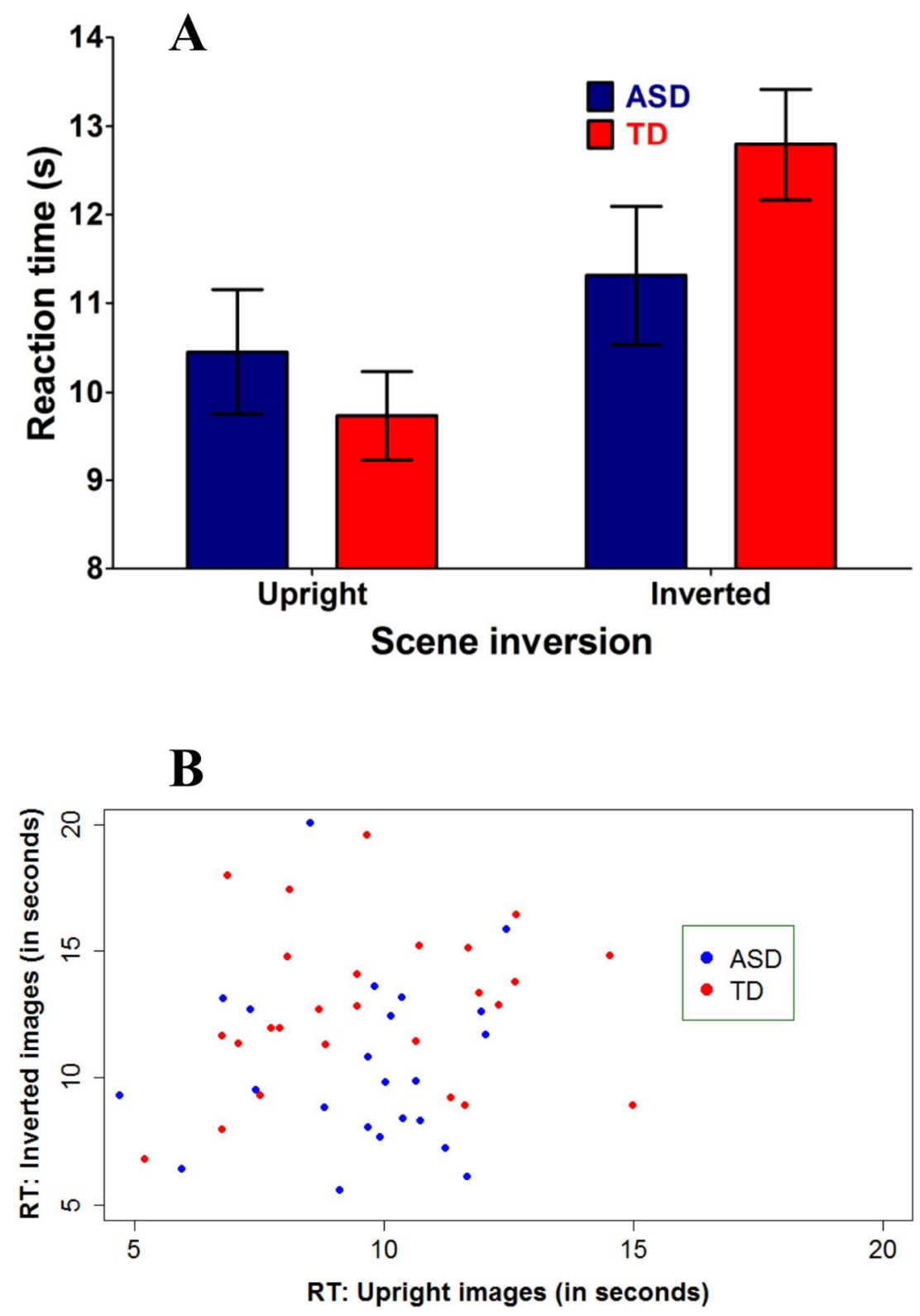

Figure 2. Overview of the RT data in the change blindness task. In (A), the data are represented as the mean performance across participants, with error bars depicting the standard error of the mean (SEM). In (C), the data are represented as the individual performance of each participant in a scatter plot, with the upright images on the abscissa and the inverted images on the ordinate. In all panels, adolescents with ASD were depicted in blue and TD adolescents in red.

$$
104 \times 150 \mathrm{~mm} \text { ( } 300 \times 300 \text { DPI) }
$$



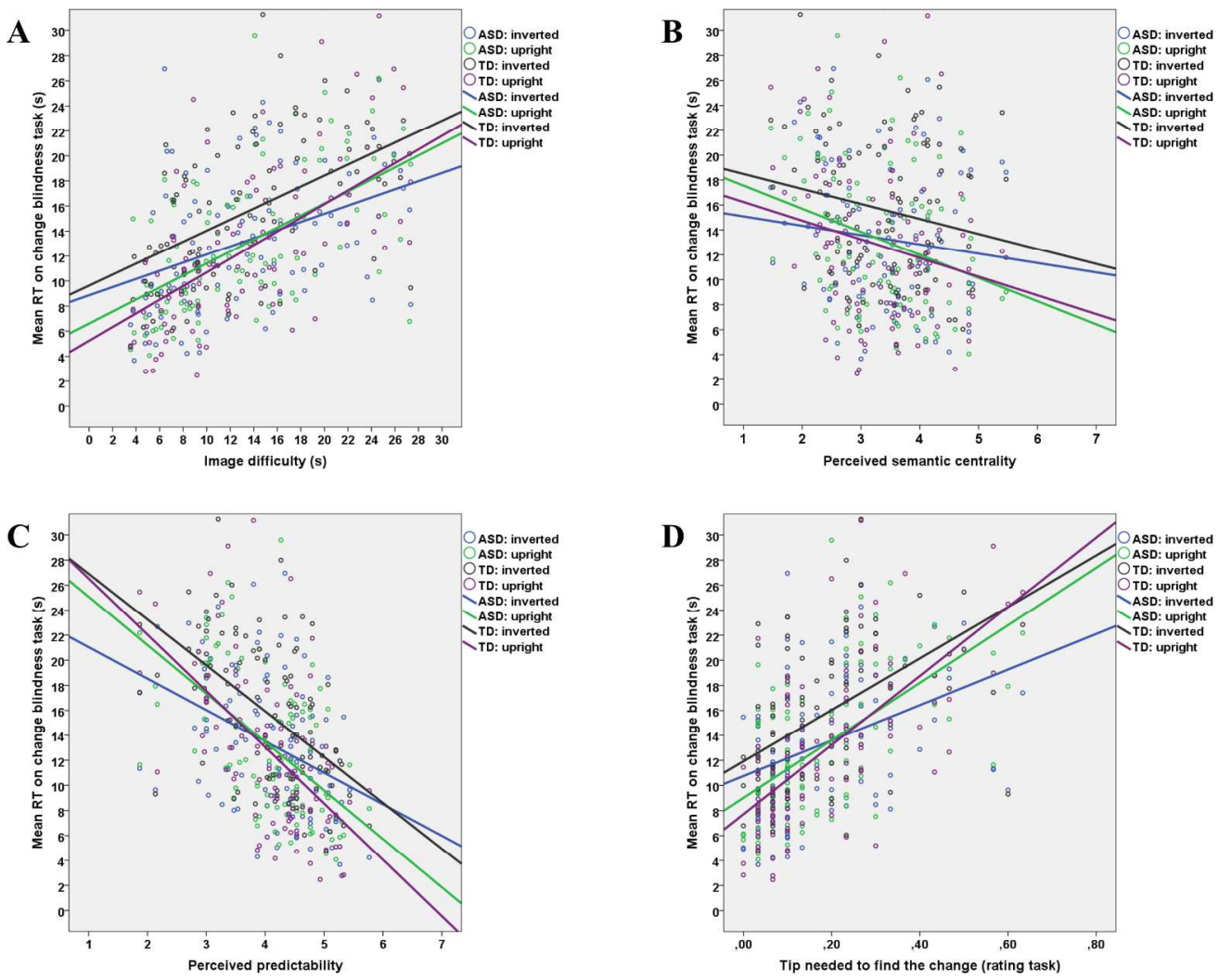

Figure 3. Visualization of the data by means of scatterplots. The mean RT per image was indicated on the ordinate axis, while in (A) we depicted the image difficulty (in mean RT), in (B) the rated semantic centrality, in (C) the rated predictability and in (D) the proportion of people requesting a 'clue' to find the change in the rating task on the abscissa. In all panels, the performance of adolescents with ASD in the upright condition was depicted in green and their performance in the inverted condition in blue. Simultaneously, the performance of the TD adolescents in the upright condition was depicted in purple and their performance in the inverted condition in dark grey.

$229 \times 183 \mathrm{~mm}(300 \times 300 \mathrm{DPI})$ 